\title{
Effects of Discretization on the K-Width of Series Elastic Actuators
}

\author{
Dylan P. Losey, Student Member, IEEE, and Marcia K. O'Malley, Senior Member, IEEE
}

\begin{abstract}
Rigid haptic devices enable humans to physically interact with virtual environments, and the range of impedances that can be safely rendered using these rigid devices is quantified by the Z-Width metric. Series elastic actuators (SEAs) similarly modulate the impedance felt by the human operator when interacting with a robotic device, and, in particular, the robot's perceived stiffness can be controlled by changing the elastic element's equilibrium position. In this paper, we explore the K-Width of SEAs, while specifically focusing on how discretization inherent in the computer-control architecture affects the system's passivity. We first propose a hybrid model for a single degree-of-freedom (DoF) SEA based on prior hybrid models for rigid haptic systems. Next, we derive a closed-form bound on the K-Width of SEAs that is a generalization of known constraints for both rigid haptic systems and continuous time SEA models. This bound is first derived under a continuous time approximation, and is then numerically supported with discrete time analysis. Finally, experimental results validate our finding that large pure masses are the most destabilizing operator in human-SEA interactions, and demonstrate the accuracy of our theoretical K-Width bound.
\end{abstract}

\section{INTRODUCTION}

During physical human-robot interaction (pHRI) it is often desirable for the robotic system to render a virtual environment, and, in particular, a virtual stiffness, with which the human will interact. Virtual stiffnesses can be used to guide humans along a pre-defined path or provide haptic feedback from simulated objects, with applications in robotic rehabilitation [1] and surgery [2]. So as to provide suitable levels of path guidance and display a variety of realistic objects, we would like to maximize the range of virtual stiffnesses that our robotic system can render. We must first ensure that these virtual stiffnesses are safe for the human, however, and will not lead to instability during pHRI.

Passivity of the robotic system guarantees pHRI stability, since (a) two passive systems coupled together result in a stable system and (b) human operators can be assumed to be passive [3]. Accordingly, we define K-Width as the range of virtual stiffnesses which a robotic system can render passively. Prior research by Colgate and Brown [4] introduced Z-Width, which generalizes K-Width to include virtual impedances (virtual stiffness and damping). Closedform constraints on the Z-Width and K-Width of a rigid haptic device controlled by a computer interface are derived in [4]-[6]. Interestingly, these works found that passivity is affected by the computer interface-a ubiquitous component in robotic systems. The computer interface operates in discrete time, while the haptic device operates in continuous

This work was funded in part by the NSF GRFP-1450681.

The authors are with the Mechatronics and Haptic Interfaces Laboratory, Department of Mechanical Engineering, Rice University, Houston, TX 77005. (e-mail: dlosey@ rice.edu) time; therefore, the computer interface must convert from a continuous signal into a discrete signal, and then back to a continuous signal. We refer to this process as discretization, and point out that discretization is known to limit the $\mathrm{K}$ Width of rigid haptic devices [4]-[6].

More recently, compliant haptic devices_-such as series elastic actuators-have emerged as a desirable platform for pHRI. Originally proposed in [7], SEAs incorporate a compliant element of known stiffness between the actuator and load, and, because of this physical compliance, SEAs fundamentally improve the robot's reflected dynamics [8]. Additionally, by measuring the spring displacement between actuator and load, we can use SEAs to straightforwardly control interaction forces between the human and robot. Several groups have explored the K-Width of SEAs [9][12], notably concluding that, for prominent linear control schemes modeled in continuous time, the stiffness of the virtual environment must be less than or equal to the stiffness of the physical spring. Research on SEA K-Width, however, has not explicitly considered discretization [13].

In this paper we extend the study of K-Width from rigid haptic devices to SEAs, and explicitly consider the effects of discretization caused by the computer interface. By accurately determining SEA K-Width, we can guarantee that SEAs will safely render virtual stiffnesses within pHRI applications such as rehabilitation robotics [14]. In Section II we rigorously define the class of systems we will consider, and create a hybrid model for SEAs which parallels the prior hybrid model for rigid haptic systems. Next, in Section III, we derive and numerically verify a closed-form bound on the K-Width of SEAs, where the resultant expression can be seen as a generalization of previous work on both rigid haptic systems and continuous time SEA models. We show that large pure masses are the most destabilizing operator, and thus, in Section IV, we validate the proposed K-Width bound through experiments on a 1-DoF SEA coupled to a pure mass. Our overall findings are summarized in Section V.

\section{Problem Statement}

Our goal is to determine the K-width of a 1-DoF SEA while considering discrete sampling and the zero-order hold imposed by the computer interface. Inspired by the work of Colgate and Brown [4], a closed-form bound on virtual stiffness will be derived as a function of the system parameters.

\section{A. Hybrid Model}

As shown in Fig. 1, we consider a 1-DoF SEA with mass $m$, viscous damping $b$, and spring stiffness $k$. The actuator position is denoted by $x_{A}$ while the load position is denoted 


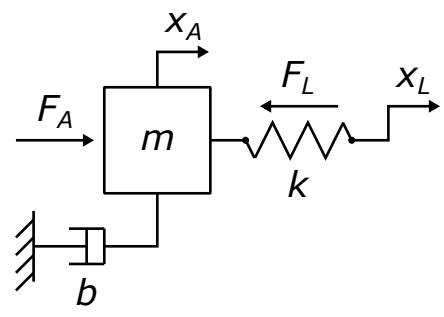

Fig. 1. Schematic of a 1-DoF SEA. The actuator and load are connected by a compliant element with constant stiffness $k$.

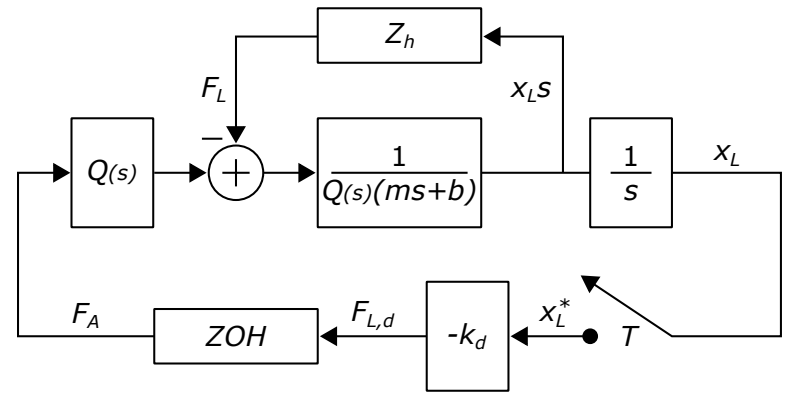

Fig. 2. Hybrid model of a 1-DoF SEA system. $Z_{h}$ is the impedance of the human operator, $x_{L}^{*}$ is the sampled load position, $\mathrm{ZOH}$ is the zero-order hold, and $Q$ is the second-order linear filter from (4).

by $x_{L}$. This SEA is acted upon by two forces-the force applied by the actuator, $F_{A}$, and the force applied by a human operator at the load, $F_{L}$. Following the convention of [8], we define $F_{L}$ as positive when the spring is being compressed. Thus, the SEA equation of motion in the time domain is

$$
\begin{gathered}
F_{A}(t)-F_{L}(t)=m \ddot{x}_{A}(t)+b \dot{x}_{A}(t) \\
F_{L}(t)=k\left(x_{A}(t)-x_{L}(t)\right)
\end{gathered}
$$

Within the Z-width literature for rigid haptic devices, where actuator and load positions are equivalent, $x_{A}$ in (1) has been replaced by $x_{L}$ (for examples, see [4]-[6]). To rewrite (1) into a form relating $F_{A}, F_{L}$, and $x_{L}$, we can combine (1) and (2) to obtain the Laplace domain equation of motion

$$
Q(s) F_{A}-F_{L}=Q(s)(m s+b) x_{L} s
$$

where $Q$ is a second-order linear filter

$$
Q(s)=\frac{k}{m s^{2}+b s+k}
$$

Throughout this work, we will find that $Q$ distinguishes our SEA system from rigid haptic devices. For now, we notice from (4) that $Q(j \omega)$ approaches 1 at low frequencies $(\omega \rightarrow$ $0)$, while $Q(j \omega)$ approaches 0 at high frequencies $(\omega \rightarrow \infty)$. Moreover, for a rigid haptic device where $k \rightarrow \infty$, we have that $Q=1$ and, by comparing (3) to (1), $x_{A}=x_{L}$.

We would like to control the SEA described by (3) such that it renders a desired stiffness, $k_{d}$, to the human operator. When controlling the continuous time SEA using a computer interface, (a) sampling is used to obtain a discrete measurement of $x_{L}$, (b) the desired load force, $F_{L, d}$, is calculated by the computer at each sample time, and then (c) a zero-order hold converts $F_{L, d}$ into $F_{A}$, where $F_{A}$ is held constant between samples. Following the notation employed by [6], we accordingly define the desired load force to be

$$
F_{L, d}(h T)=-k_{d} x_{L}(h T) \quad \forall h \in \mathbb{N}
$$

where $h$ is the discrete time variable and $T$ is the sample period. For convenience, we also introduce the sampling frequency, $f_{s}$, which is defined as $f_{s}=1 / T$. As explained by [3], in the Z-width literature no distinction is typically made between the virtual environment and the control law; hence, we will treat the desired load force as our commanded actuator force, where the merits of this decision are discussed in Section II-B. Therefore, we define the actuator force as

$$
F_{A}(t)=F_{L, d}(h T) \quad \forall t \in[h T ;(h+1) T[, h \in \mathbb{N}
$$

noting that (6) captures the effects of the zero-order hold. To summarize, the block diagram of our SEA system described by (3), (5), and (6) is shown in Fig. 2. We note that Fig. 2 is the same as the analogous block diagram for rigid haptic devices shown in [4] when $Q=1$.

\section{B. Controller Selection}

It is standard practice when studying Z-width to use the desired load force as the commanded actuator force [4][6], [15]-[18], like we have done in (6). Within the SEA literature, however, cascaded force-velocity control [9]-[11] is commonly used to determine $F_{A}$ based on $F_{L, d}$. To demonstrate that (6) is still a "good" choice of control law, we will briefly show that our system also captures several salient features of cascaded force-velocity controlled SEAs. Consider the case without discretization $(T \rightarrow 0)$, such that (5) and (6) simplify to $F_{A}(t)=F_{L, d}(t)=-k_{d} x_{L}(t)$, and define the impedance of the SEA at the load, $Z_{L}$, to be $Z_{L}=-F_{L} / \dot{x}_{L}$. From Fig. 2, we can now write

$$
\lim _{T \rightarrow 0} Z_{L}(s)=\frac{k}{s}\left(\frac{m s^{2}+b s+k_{d}}{m s^{2}+b s+k}\right)
$$

If the phase of the parenthesized part of (7) becomes negative, then the phase of the impedance transfer function will drop below $-90^{\circ}$, and the SEA system becomes non-passive [9]. Hence, we have that $k_{d} \leq k$ is a necessary condition for passivity when discretization is not considered, as was previously derived for cascaded force-velocity controllers [9]-[11]. Next, using (4), we can rewrite (7) more concisely

$$
\lim _{T \rightarrow 0} Z_{L}(s)=\frac{k}{s}+\left(\frac{k_{d}-k}{s}\right) Q(s)
$$

Recalling the frequency response of $Q(j \omega)$, we observe that, when discretization is not considered, $Z_{L}(j \omega)$ approaches the desired spring stiffness at low frequencies, where $Q(j \omega) \rightarrow 1$, and $Z_{L}(j \omega)$ approaches the physical spring stiffness at high frequencies, where $Q(j \omega) \rightarrow 0$. The same result that was also found with cascaded force-velocity control [11]. Therefore, we conclude that (6) is a "good" choice of control law, both in that it parallels prior Z-width research, and in that it captures some important aspects of more common SEA controllers. 


\section{PASSIVITy RESUlts}

Using the system described in Section II, we now seek to derive and evaluate the K-width which ensures safe operation of an SEA. Because the spring introduces $Q$ into (3), our equation of motion is best suited for analysis in the frequency domain. Unfortunately, the time domain approaches developed for rigid haptic systems by Abbott and Okamura [5] and Diolaiti et al. [6] are therefore not well suited for our application. Earlier work by Colgate and Schenkel [19] leverages frequency domain techniques; however, their method does not readily incorporate $Q$. Hence, in this section we first create a continuous approximation of our hybrid system, from which the passivity constraint can be derived using linear analysis techniques commonly applied to SEAs [9]-[12]. We then support the derived K-width using a discrete model, with which coupled stability can be numerically evaluated [15]-[18]. Our resultant approach is perhaps most similar to Colonnese and Okamura [16], in that we will consider both continuous and discrete models; however, unlike [16], we here claim to find a closed-form passivity constraint.

\section{A. Continuous Approximation}

In order to write the hybrid model shown in Fig. 2 as a continuous system, we need a continuous approximation of (6), the zero-order hold. As described by Adams and Hannaford [3], the zero-order hold can be approximated as a low-pass filter, where the filter's time constant, $\tau$, is half of the sampling time

$$
Z O H(s)=\frac{1}{(\tau s+1)}, \quad \tau=\frac{T}{2}
$$

This approximation, however, is only valid when aliasing effects due to sampling are negligible [3]. Accordingly, we assume that the system's sampling frequency, $f_{s}$, exceeds twice the natural frequency of the SEA, $f_{n}$. Since we will later show that $k_{d} \leq k$ is a necessary condition for passivity of the hybrid system, the maximum natural frequency of the SEA should be $f_{n}=\sqrt{k / m} \mathrm{rad} / \mathrm{s}$ [8]. In other words, our assumption amounts to the following lower bound on sampling frequency (measured in $\mathrm{Hz}$ )

$$
f_{s}>\frac{1}{\pi} \sqrt{\frac{k}{m}}
$$

This constraint is similar to the bound found for rigid haptic systems in [6], except that $k_{d}$ has been here replaced by $k$. Now applying (9), we can derive from Fig. 2 the impedance transfer function for the SEA system, $Z_{L}$, which relates input load velocity to opposing load force

$$
Z_{L}(s)=\frac{F_{L}}{-x_{L} s}=\frac{k}{s} \cdot \frac{\left(m s^{2}+b s\right)(\tau s+1)+k_{d}}{\left(m s^{2}+b s+k\right)(\tau s+1)}
$$

\section{B. Impedance Passivity}

The SEA system is passive if the continuous approximation of its impedance transfer function (11) is positive real. Relevant necessary and sufficient conditions for positive realness include: (a) poles of $Z_{L}$ on the imaginary axis must be simple, (b) $Z_{L}$ must be stable, and (c) the real part of $Z_{L}$ must be nonnegative everywhere along the $j \omega$ axis, except for poles [20]. Condition (a) is satisfied since the only pole on the imaginary axis occurs at the origin. We can verify (b) using the Hurwitz stability criterion; noting that the coefficients of the denominator of $Z_{L}$ are positive, we obtain the following requirement for stability from the Hurwitz determinates [21]

$$
b\left(m+b \tau+k \tau^{2}\right)>0
$$

Because $m, b, k$, and $\tau$ are necessarily positive, we therefore have that condition (b) is always satisfied. Finally, we can test condition (c) by defining $Z_{L}(s)=B(s) / A(s)$. Then

$$
\operatorname{Re}\left\{Z_{L}(j \omega)\right\}=\operatorname{Re}\left\{\frac{B(j \omega)}{A(j \omega)}\right\}=\operatorname{Re}\left\{\frac{B(j \omega) A(-j \omega)}{A(j \omega) A(-j \omega)}\right\}
$$

where the final denominator is nonnegative [20]. Based on this result, if $\operatorname{Re}\{B(j \omega) A(-j \omega)\} \geq 0$, we can conclude that condition (c) is satisfied, and thus $Z_{L}$ is positive real.

For ease of notation, let's define a polynomial $P$ to be

$$
P(\omega)=\sum_{i} d_{i} \omega^{i}=\operatorname{Re}\{B(j \omega) A(-j \omega)\}
$$

Plugging (11) into (14), all coefficients $d_{i}$ of the polynomial $P$ are zero, except for

$$
\begin{gathered}
d_{4}=k \tau\left(b k \tau+m k_{d}\right) \\
d_{2}=k\left(b k-b k_{d}-k k_{d} \tau\right)
\end{gathered}
$$

Coefficient $d_{4}$ is always positive due to positive parameters, and dominates the summation in (14) at higher frequencies. Coefficient $d_{2}$, however, is only nonnegative when

$$
b\left(k-k_{d}\right) \geq \frac{k k_{d} T}{2}
$$

We conclude that (16) is the necessary and sufficient condition to guarantee that (14) is nonnegative at all frequencies, and, by construction, ensure that the SEA system is passive. A plot of (16) evaluated at different damping values and sampling frequencies is shown in Fig. 3.

\section{Comparison to Existing $K$-Width Constraints}

We have found that the SEA system described in Section II is guaranteed to be passive when rendering a virtual spring if and only if (16) is satisfied. Next, we would like to compare this constraint to analogous passivity constraints derived for rigid haptic devices [4]-[6], as well as for SEA systems when discretization is not considered [9]. When $k \rightarrow \infty$ and $T>$ 0 , we know from Section II-A that our SEA system mimics a rigid haptic device with discretization. In this case, because of L'Hôpital's rule, (16) simplifies to

$$
b \geq \frac{k_{d} T}{2}
$$

which matches the result found in [4]-[6] specifically for rigid systems with no virtual damping, sensor quantization, or Coulomb friction. On the other hand, when $k$ is finite 


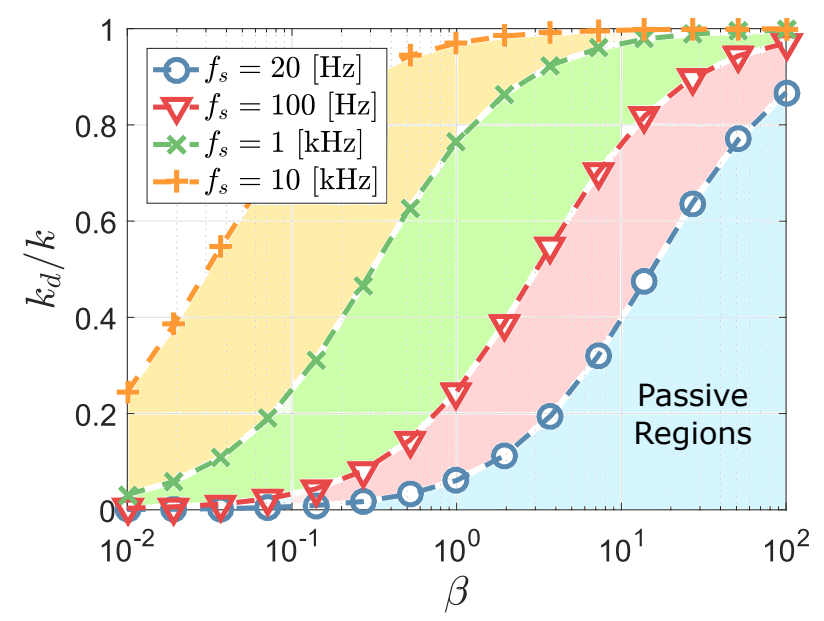

Fig. 3. Normalized SEA damping $(\beta)$ vs. the normalized stiffnesses $\left(k_{d} / k\right)$ which can be rendered passively at different sampling frequencies. $\beta$ is defined as an independent variable $b$ divided by $1.75 \mathrm{~N} \cdot \mathrm{s} / \mathrm{m}$, the estimated damping of our hardware. Passivity regions (total area below each line) were found by evaluating (16), with $k$ taken from Table I. Increasing the physical damping and/or sampling frequency increases the $k_{d}$ which can be passively rendered.

and $T \rightarrow 0$, we have a compliant SEA system without discretization. In this case, (16) simplifies to

$$
k_{d} \leq k
$$

which is the same as the result reported by [9] when $F_{A}=$ $F_{L, d}$. Accordingly, we claim that (16) is a generalization of (17) and (18), providing the passivity constraint for rendering a virtual wall while considering both the actuatorload connection stiffness and the presence of discretization.

\section{Passivity vs. Coupled Stability}

To further support the passivity constraint derived above, we next consider an entirely discrete model of our SEA system. This discrete model will be used to determine the maximum virtual stiffness which results in stability when the SEA is coupled to a human operator of known impedance. Importantly, because the discrete model's stability bounds are the same as the stability bounds of the hybrid system [16], no approximations are necessary here. We will, however, need to choose $Z_{h}$, the impedance of the human operator. Let $Z_{h, 1}$ be the impedance of a pure mass attached to the load, which has been shown to be the most destabilizing operator for our SEA system when $T \rightarrow 0$ [9]. Additionally, let $Z_{h, 3}$ and $Z_{h, 5}$ be three- and five-parameter impedance models of a human interacting with a haptic device as described by [22] and [23], respectively. Thus, we have three choices for $Z_{h}$

$$
\begin{gathered}
Z_{h, 1}(s)=m_{h} s \\
Z_{h, 3}(s)=\frac{m_{h} s^{2}+b_{h} s+k_{h}}{s} \\
Z_{h, 5}(s)=\frac{m_{h} b_{g} s^{3}+\left(m_{h} k_{g}+b_{g} b_{h}\right) s^{2}+\rho s+k_{g} k_{h}}{m_{h} s^{3}+\left(b_{h}+b_{g}\right) s^{2}+\left(k_{h}+k_{g}\right) s}
\end{gathered}
$$

where $\rho=\left(k_{g} b_{h}+b_{g} k_{h}\right)$. The mass, damping, and stiffness parameter values we used for these models are taken from [9], [22], and [23], and are listed in Table I.

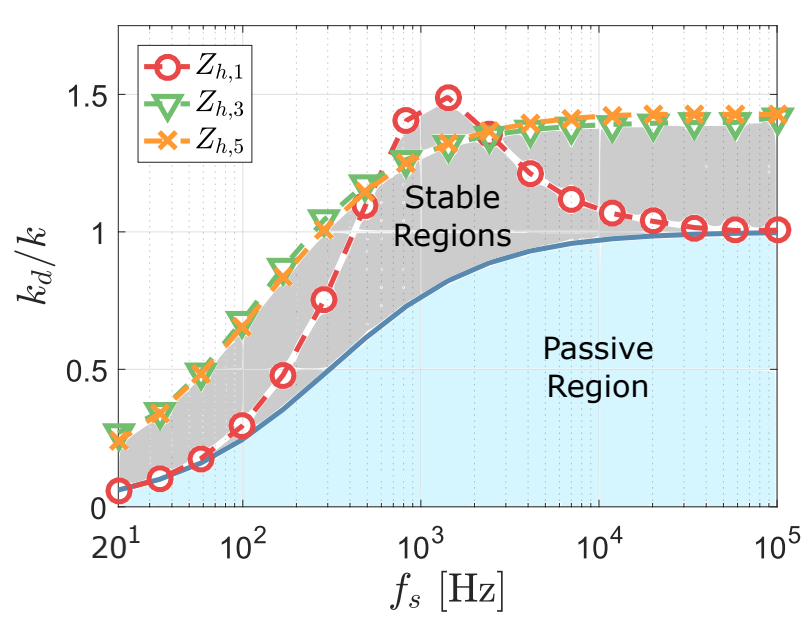

Fig. 4. Passivity and coupled stability regions as a function of sampling frequency using (16), (22), and parameters from Table I. Normalized stiffness values below each dashed line are stable when our SEA is coupled to the corresponding human impedance model $\left(Z_{h, i}\right)$. Normalized stiffness values beneath the solid line can be rendered passively.

Now that we have defined $Z_{h}$, we can find the continuous transfer function which relates actuator force to load position. Referring to the block diagram in Fig. 2, we obtain

$$
G_{i}(s)=\frac{x_{L}}{F_{A}}=\frac{1}{m s^{2}+b s+s Q^{-1}(s) Z_{h, i}(s)}
$$

where subscript $i$ indicates the choice of impedance model from (19). Again, we notice that $Q$ distinguishes (20) from the equivalent expression found for rigid haptic devices [17], [19], where $Q=1$. Next, following the process employed by [15]-[18], the zero-order hold and $G_{i}(s)$ are converted into a discrete transfer function using $\mathcal{Z}$, the $\mathcal{Z}$-transform operator. Applying this discrete transfer function, the characteristic equation of the coupled system becomes

$$
1+k_{d} \mathcal{Z}\left[\mathrm{ZOH} G_{i}(s)\right]=0
$$

And so, by definition, the values of $k_{d}$ which yield coupled stability must satisfy the constraint

$$
k_{d}<\operatorname{GM}\left(\mathcal{Z}\left[\mathrm{ZOH} G_{i}(s)\right]\right)
$$

where $\operatorname{GM}(\cdot)$ denotes the gain margin. In practice, the right side of (22) can be numerically solved in MATLAB.

Using (22), we calculated the upper bound on $k_{d}$ for coupled stability when considering each of the human impedance models given by (19). We then plotted these results in Fig. 4, along with the passivity region defined by (16). From the resultant plot, we observed that our passivity region is contained within each of the coupled stability regions, as should be expected. Perhaps more surprisingly, we also found that a pure mass attached at the load $\left(Z_{h, 1}\right)$ is not the "worst-case"-or most destabilizing operator-at all sampling frequencies. Indeed, while the coupled stability bound for $m_{h}=0.1 \mathrm{~kg}$ matched the passivity bound when $f_{s}$ was greater than $10 \mathrm{kHz}$ or less than $100 \mathrm{~Hz}$, between these sampling frequencies the stable $k_{d}$ region noticeably exceeded the passive $k_{d}$ region. To better understand this 


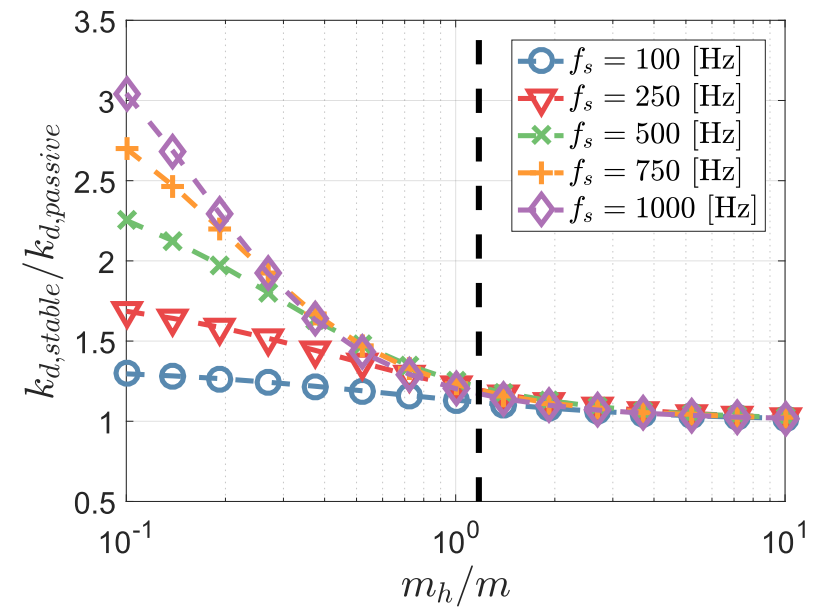

Fig. 5. Effect of human mass $\left(m_{h}\right)$ on the ratio between coupled stability bounds and passivity bounds at different sampling frequencies. $k_{d, \text { stable }}$ is defined as the upper bound of (22) when considering $Z_{h, 1}$, the impedance model for a pure mass attached to the load. $k_{d, p a s s i v e}$ is defined as the upper bound of (16). With the exception of the independent variable $m_{h}$, all parameters were taken from Table I. The vertical dashed line shows the value of $m_{h} / m$ used during our subsequent experiments.

result, we next reapplied (22) with $Z_{h, 1}$ while testing a variety of values for $m_{h}$, the human mass. A plot of our findings is shown in Fig. 5, where $k_{d \text {,stable }}$ is the upper bound of the coupled stability region, and $k_{d, \text { passive }}$ is the upper bound of the passive region. Based on this plot, it is clear that as $m_{h}$ increases, the coupled stability region converges to the passivity region regardless of sampling frequency; hence, we conclude that large pure masses (for example, $\left.m_{h} / m>3\right)$ are the most destabilizing operator for our SEA system when $T>0$. Moreover, the fact that $k_{d, \text { stable }}$ converges to $k_{d, p a s s i v e}$ supports the passivity constraint (16).

\section{EXPERIMENTS}

To validate our passivity and coupled stability results derived in Section III, we next conducted experiments using the linear SEA described by [12], which is shown in Fig. 6. The actuator consisted of a brushed DC motor (Maxon Motor, RE 30) and rotary incremental encoder (Maxon Motor, HEDL 5540) attached by capstan drive to a translational slider, whose position was denoted as $x_{A}$. A bidirectional spring was connected to the output of this translational slider, and the spring's displacement, $\Delta x=x_{A}-x_{L}$, was directly measured using a linear incremental encoder (US Digital, EM1-0-500-I). By subtracting the spring displacement from the slider position, $x_{A}-\Delta x$, we were able to measure $x_{L}$, the position of the load. The actuator's mass, $m$, and viscous damping, $b$, were identified using the logarithmic decrement method and then verified with linear regression, while the spring stiffness, $k$, was found by applying known forces and measuring displacement; these identified SEA parameters are listed in Table I. Control was implemented on a desktop PC using Simulink (MathWorks) with the QUARC blockset (Quanser). The computer interfaced with the SEA hardware through a Q8-USB data acquisition device (Quanser), and had a maximum sampling frequency of $1 \mathrm{kHz}$.
TABLE I

PHySicAl PARAMETERS FOR OUR 1-DoF SEA AND HUMAN OPERATOR IMPEDANCE MODELS

\begin{tabular}{lcccc} 
Parameter & Variable & \multicolumn{3}{c}{ Value } \\
\hline SEA Mass [kg] & $m$ & & 0.375 & \\
SEA Damping [N·s/m] & $b$ & & 1.75 & \\
SEA Stiffness [N/m] & $k$ & & 1075 & \\
\hline Impedance Model & & $Z_{h, 1}$ & $Z_{h, 3}$ & $Z_{h, 5}$ \\
Source & & {$[9]$} & {$[22]$} & {$[23]$} \\
\hline Human Mass [kg] & $m_{h}$ & 0.1 & 0.15 & 1.46 \\
Human Damping [N.s/m] & $b_{h}$ & - & 4.8 & 4.5 \\
Human Stiffness [N/m] & $k_{h}$ & - & 600 & 48.8 \\
Grasp Damping [N.s/m] & $b_{g}$ & - & - & 7.9 \\
Grasp Stiffness [N/m] & $k_{g}$ & - & - & 375
\end{tabular}

During experiments, we varied the sampling frequency of our computer interface, $f_{s}$, while rendering different values of virtual stiffness, $k_{d}$. Since the passivity of an SEA can be difficult to observe, we instead tested for coupled stability when a pure mass, $m_{h}$, was attached to the load, i.e., $Z_{h, 1}$ in (19). Based on our discussion of Fig. 5 in Section III-D, we know that large pure masses are the most destabilizing operators for our SEA system, and thus, when a sufficiently large mass is attached at the load, the coupled stability region approximates the passivity region. Accordingly, to get an idea of the $k_{d}$ that can be rendered passively, we set $m_{h}=$ $0.441 \mathrm{~kg}$, noting that $m_{h}$ could not be further increased in our system due to actuator saturation and maximal spring displacement. Hence, during our experiments $m_{h} / m \approx 1.18$, which unfortunately is less than the $m_{h} / m>3$ ratio we recommended in Section III-D; as a result, the stability region will be noticeably larger than the passivity region (as observed in Fig. 7). For a given $f_{s}$ and $k_{d}$, a malicious human operator perturbed the load mass by briefly applying an impulse force and then abruptly releasing, in an attempt to cause the coupled SEA and load mass to become unstable. If the oscillations of $x_{L}$ decreased in magnitude, $k_{d}$ was increased and the experiment was repeated; otherwise, if the oscillations of $x_{L}$ became unstable, $k_{d}$ was recorded and we started to test the next sampling frequency.

The results of our experiments can be seen in Fig. 7. The values of normalized stiffness, $k_{d} / k$, which are predicted to be stable (below dashed line) were found by evaluating (22) with $Z_{h, 1}$, where here $m_{h}=0.441 \mathrm{~kg}$. The actual values of normalized stiffness at which our SEA system became unsta-

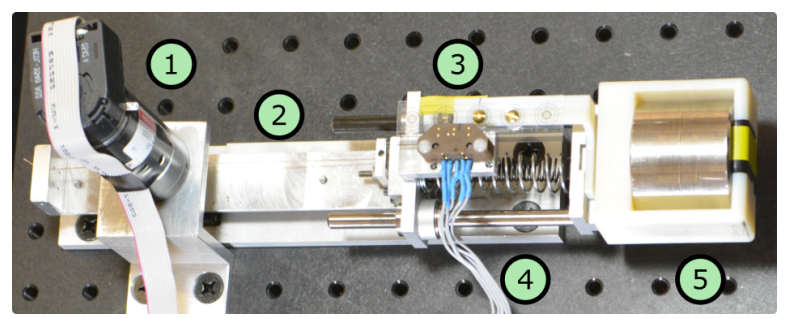

Fig. 6. 1-DoF SEA hardware used for our experiments. (1) motor and rotary encoder, (2) translational slider with position $x_{A}$, (3) linear encoder measuring spring displacement, (4) spring, (5) load mass with position $x_{L}$. 


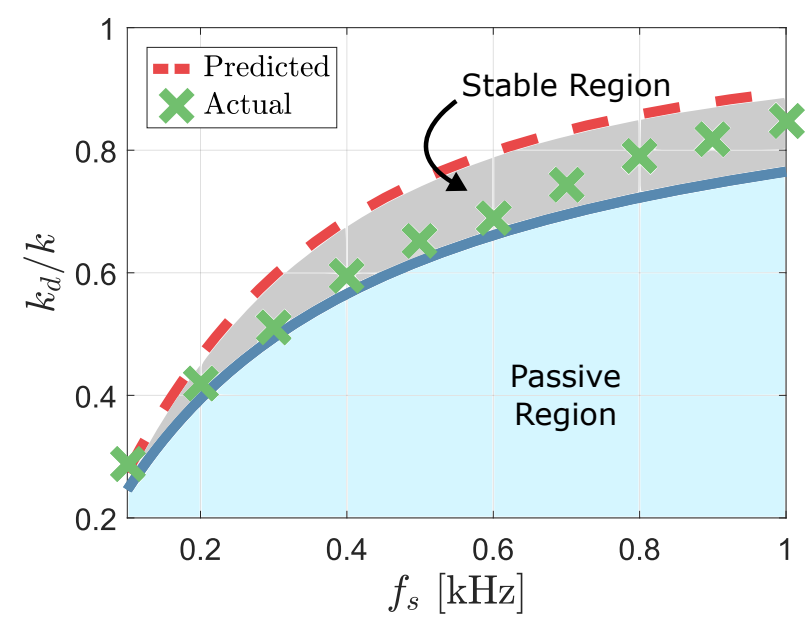

Fig. 7. Experimental results. A load mass was attached to the SEA and then perturbed by a malicious operator. The lowest normalized stiffness, $k_{d} / k$, at which oscillations of the load mass became unstable is plotted as a function of sampling frequency, $f_{s}$. Predicted values for coupled stability and the passivity region were obtained using the load mass, $m_{h}=0.441$ $\mathrm{kg}$, and the identified SEA parameters listed in Table I.

ble are marked. We observe that the experimental coupled stability region is contained within the theoretical coupled stability region, and, on average, there is a $9.98 \%$ error between the experimental and theoretical values for $k_{d, \text { stable }}$. We also show the passivity region (below solid line), which was defined by (16). Because the coupled stability and passivity regions are here similar, we will approximate stability as passivity; therefore, we claim that the values of $k_{d}$ where our SEA became unstable approximate the maximum virtual stiffnesses that can be rendered passively. Following this line of reasoning, the experimental passivity region is slightly larger than the theoretical passivity region, with a $7.85 \%$ average error for $k_{d, \text { passive }}$.

\section{CONCLUSION}

We have found the K-Width of SEAs-a class of compliant haptic devices-while explicitly considering discrete sampling and the zero-order hold imposed by the computer interface. We first described the hybrid model for a 1DoF SEA, from which we obtained $Q$, a linear filter that distinguishes SEAs from rigid haptic devices. After making a continuous approximation of the zero-order hold, we applied the positive realness test, and derived a closed-form bound on SEA K-Width that generalizes previous work on both rigid haptic systems and continuous SEA models. To numerically verify this bound, we next considered a discrete model of our SEA coupled to human operators of known impedance. Interestingly, we found that large pure masses are the most destabilizing operator, and thus, when an SEA attempting to render a virtual stiffness is coupled to a large pure mass, the coupled stability region matches the passivity region. We leveraged this similarity between coupled stability and passivity during our experiments, where we confirmed that the SEA K-Width could be predicted by our theoretical closedform bound. Our findings can be used to determine the virtual stiffnesses that can be safely rendered by an SEA, such as for rehabilitation applications. These findings can also be applied to refine the K-Width of other haptic devicesbesides SEAs-which have non-negligible compliance.

\section{REFERENCES}

[1] A. Duschau-Wicke, J. von Zitzewitz, A. Caprez, L. Lunenburger, and R. Riener, "Path control: A method for patient-cooperative robot-aided gait rehabilitation," IEEE Trans. Neural Syst. Rehabil. Eng., vol. 18, no. 1 , pp. $38-48,2010$.

[2] S. A. Bowyer, B. L. Davies, and F. R. y Baena, "Active constraints/virtual fixtures: A survey," IEEE Trans. Robot., vol. 30, no. 1, pp. 138-157, 2014.

[3] R. J. Adams and B. Hannaford, "Stable haptic interaction with virtual environments," IEEE Trans. Robot. Autom., vol. 15, no. 3, pp. 465474, 1999.

[4] J. E. Colgate and J. M. Brown, "Factors affecting the z-width of a haptic display," in Proc. IEEE Conf. Robot. Autom., 1994, pp. 32053210 .

[5] J. J. Abbott and A. M. Okamura, "Effects of position quantization and sampling rate on virtual-wall passivity," IEEE Trans. Robot., vol. 21, no. 5, pp. 952-964, 2005.

[6] N. Diolaiti, G. Niemeyer, F. Barbagli, and J. K. Salisbury, "Stability of haptic rendering: Discretization, quantization, time delay, and coulomb effects," IEEE Trans. Robot., vol. 22, no. 2, pp. 256-268, 2006.

[7] G. A. Pratt and M. M. Williamson, "Series elastic actuators," in Proc. IEEE/RSJ Conf. Intelligent Robots Syst., 1995, pp. 399-406.

[8] D. W. Robinson, "Design and analysis of series elasticity in closedloop actuator force control," Ph.D. dissertation, Dept. Mech. Eng., MIT, Cambridge, MA, 2000

[9] F. Sergi and M. K. O'Malley, "On the stability and accuracy of high stiffness rendering in non-backdrivable actuators through series elasticity," Mechatronics, vol. 26, pp. 64-75, 2015.

[10] N. L. Tagliamonte and D. Accoto, "Passivity constraints for the impedance control of series elastic actuators," Proc. Inst. Mech. Eng. I, J. Syst. Control Eng., vol. 228, no. 3, pp. 138-153, 2014.

[11] H. Vallery, J. Veneman, E. Van Asseldonk, R. Ekkelenkamp, M. Buss, and H. Van Der Kooij, "Compliant actuation of rehabilitation robots," IEEE Robot. Autom. Mag., vol. 15, no. 3, pp. 60-69, 2008.

[12] D. P. Losey, A. Erwin, C. G. McDonald, F. Sergi, and M. K. O'Malley, "A time domain approach to control of series elastic actuators: Adaptive torque and passivity-based impedance control," IEEE/ASME Trans. Mechatronics, vol. 21, no. 4, pp. 2085-2096, 2016.

[13] A. Calanca, R. Muradore, and P. Fiorini, "A review of algorithms for compliant control of stiff and fixed-compliance robots," IEEE/ASME Trans. Mechatronics, vol. 21, no. 2, pp. 613-624, 2016.

[14] J. F. Veneman, R. Kruidhof, E. E. Hekman, R. Ekkelenkamp, E. H. Van Asseldonk, and H. Van Der Kooij, "Design and evaluation of the lopes exoskeleton robot for interactive gait rehabilitation," IEEE Trans. Neural Syst. Rehabil. Eng., vol. 15, no. 3, pp. 379-386, 2007.

[15] V. Chawda, O. Celik, and M. K. O'Malley, "A method for selecting velocity filter cut-off frequency for maximizing impedance width performance in haptic interfaces," J. Dyn. Syst. Meas. Control, vol. 137, no. 2, p. 024503, 2015.

[16] N. Colonnese and A. Okamura, "M-width: Stability and accuracy of haptic rendering of virtual mass," Robotics, vol. 41, 2013.

[17] J. J. Gil, A. Avello, A. Rubio, and J. Florez, "Stability analysis of a 1 DoF haptic interface using the Routh-Hurwitz criterion," IEEE Trans. Control Syst. Technol., vol. 12, no. 4, pp. 583-588, 2004.

[18] J. J. Gil, M. J. Puerto, I. Díaz, and E. Sánchez, "On the Z-width limitation due to the vibration modes of haptic interfaces," in Proc. IEEE/RSJ Conf. Intelligent Robots Syst., 2010, pp. 5054-5059.

[19] J. E. Colgate and G. G. Schenkel, "Passivity of a class of sampleddata systems: Application to haptic interfaces," J. Robot. Syst., vol. 14, no. 1, pp. 37-47, 1997.

[20] K. J. Åström and B. Wittenmark, Adaptive Control, 2nd ed. Mineola, NY, USA: Dover Publications, 2008.

[21] K. Ogata, Modern Control Engineering, 5th ed. Boston, MA, USA: Prentice Hall, 2010.

[22] K. J. Kuchenbecker, J. G. Park, and G. Niemeyer, "Characterizing the human wrist for improved haptic interaction," in Proc. ASME Mech. Eng. Congress and Exposition, 2003, pp. 591-598.

[23] J. E. Speich, L. Shao, and M. Goldfarb, "Modeling the human hand as it interacts with a telemanipulation system," Mechatronics, vol. 15, no. 9, pp. 1127-1142, 2005. 